\title{
A systematic approach to reliably characterize soils based on Bevameter testing
}

\author{
Maximilian Apfelbeck*, Sebastian Kuß, Bernhard Rebele, Bernd Schäfer \\ DLR (German Aerospace Center), Institute of Robotics and Mechatronics, Münchner Str. 20, 82234 Weßling, Germany
}

\begin{abstract}
Although a lot of information about soil parameter identification exists in literature, there is currently no algorithm who makes use both of state of the art identification methodologies and incorporating statistical analysis. In this paper a state of the art soil parameter identification method is presented including the calculation of its standard deviations and a proper weighting of the objective function. With this algorithm and a Bevameter with advanced sensor and actuator technology a test campaign is started to find a reliable soil preparation, which is applicable to a large planetary rover performance testbed. Furthermore, the preparation method has to be valid and stable for various types of dry, granular and frictional soils, typically used for planetary rover testing in space robotics, since the result of pre-tests show that the soil parameters are highly depending on the preparation. Besides preparation, the soil parameters are also influenced by different Bevameter test setup variables. Thus, the effect of the penetration velocity as well as the penetration tool geometry for pressure-sinkage tests on soil parameters is investigated. For shear tests the influence of the dimension of the shear ring is analysed as well as the variation of the grouser height, the number of the grousers and the increase of the rotational shear velocity. The results of the extensive test campaign are evaluated by the proposed identification algorithms.
\end{abstract}

(c) 2011 ISTVS. Published by Elsevier Ltd. All rights reserved.

Keywords: Bevameter; Soil parameter; Testbed; Identification; Planetary rover; Statistical analysis

\section{Introduction}

Although the rover performance and mobility testing plays an important role in the ExoMars programme of the European Space Agency (ESA), simulation of the rover performance is an additional part for a successful mission. With a verified and validated simulation tool, special mobility cases occurring during the mission can be examined leading to the best trajectory of the vehicle. Furthermore, in an early stage of a project, simulation has the capability to provide information about the performance of different mobility concepts. An example for such a simulation tool is given in [1]. Therein, a soft soil contact model is used to describe the interaction between a wheel and a soft deformable soil [2]. To verify and validate the

\footnotetext{
* Corresponding author. Tel.: +49 815328 3526; fax: +49 8153281134.

E-mail address: maximilian.apfelbeck@dlr.de (M. Apfelbeck).
}

simulation software, testing and simulation have to go hand in hand. Therefore, the test facility described in [3] is used with the current ExoMars BB2 breadboard (see Fig. 1). An essential input for the simulations are the soil parameters identified from Bevameter measurements (see Fig. 2). However, a reliable mechanical characterization of soft soil by Bevameter testing is a very delicate issue to accomplish, since the soil parameter determination often depends to a large extent on several testing impacts, like the soil preparation method. Moreover, the identified parameters are influenced by several test setup parameters. Using soil parameters identified with improper test setup variables on a soil prepared with an unreliable preparation method as inputs for validation simulations leads to incorrect simulation results and consequently to incorrect validation and correlation results. Therefore, an applicable and stable soil preparation method is indispensable as well as information about the effect of the variation of the test 


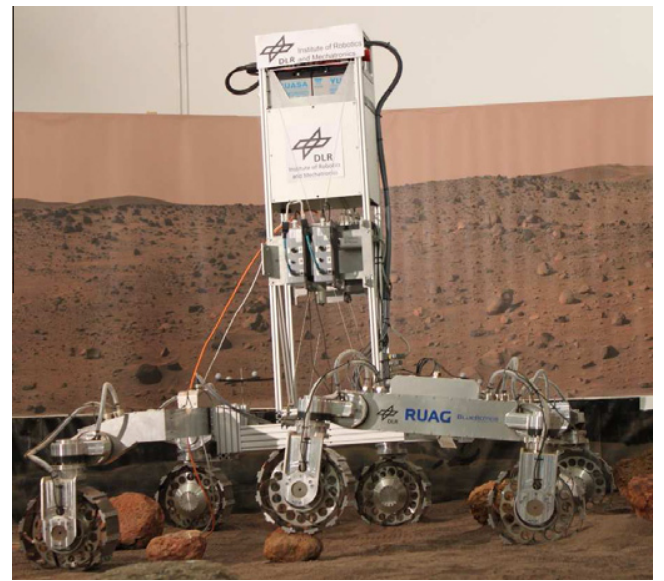

Fig. 1. ExoMars BB2 breadboard.

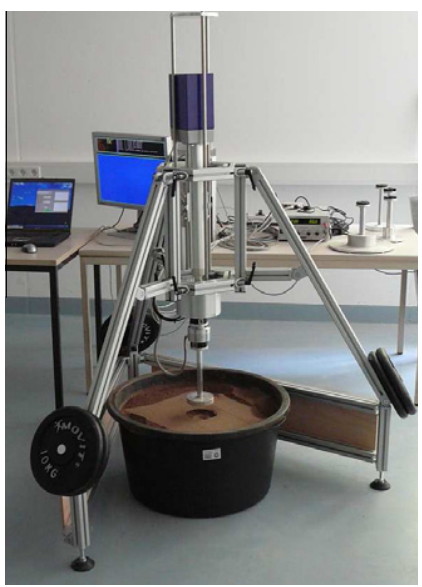

Fig. 2. Bevameter for soil characterization.

setup parameters on the soil parameters. These issues are investigated in an extensive test campaign presented in this paper. The test campaign is performed on various types of dry, granular and frictional soil, typically used for planetary rover testing in space robotics. Besides the preparation and the test setup, the identification algorithm is another important part to receive stable and reliable soil parameters. The currently known soil identification methods $[4,5]$ do neither incorporate a statistical analysis of the identified parameters nor consider the nonlinear behaviour of the soil equations directly. Both issues are included in the soil identification algorithm presented in this paper.

In Section 2 the soil parameter identification methods are described. The test results are shown in Section 3, the identification results in Section 4. Section 5 finally gives a conclusion of this paper.

\section{Soil parameter identification}

In the following section a general approach for an identification problem is given. This approach is adopted to the identification of soil parameters using a pressure-sinkage test or a shear test (see Sections 2.1 and 2.2). For parameter identification a general minimization problem is used

$$
\begin{aligned}
\min _{\hat{\theta}} E(\hat{\theta}) & =\min _{\hat{\theta}} \frac{1}{2}\left\|\mathbf{w}_{\mathbf{r}} \cdot \mathbf{e}(\mathbf{X}, \hat{\theta})\right\|^{2} \\
& =\min _{\hat{\theta}} \frac{1}{2}\left\|\mathbf{w}_{\mathbf{r}} \cdot[\mathbf{y}-\mathbf{f}(\mathbf{X}, \hat{\theta})]\right\|^{2} .
\end{aligned}
$$

The objective function is given by $E(\hat{\theta})$, and the error function by $\mathbf{e}(\mathbf{X}, \hat{\theta})$. The vector $\mathbf{y}$ consists of the measured values. The model function of the identified parameters, $\hat{\theta}$, and controlled inputs, $\mathbf{X}$, is denoted by $\mathbf{f}(\mathbf{X}, \hat{\theta})$. The controlled inputs are variables or values on which the result of the model function depends. Furthermore, a weighting factor $\mathbf{w}_{\mathbf{r}}$ is included in Eq. 1. The solver for the optimization problem depends on the chosen model function, i.e. a nonlinear solver (e.g. Levenberg-Marquardt) for a nonlinear objective function or a linear solver for a linear objective function. An overview of solvers for minimization problems is given in [6]. The quality of the fit is calculated by the root mean square of the identification error over the measured values:

$\epsilon=1-\frac{\sqrt{\frac{\sum \mathbf{e}(\mathbf{X}, \hat{\theta})^{2}}{N-2}}}{\frac{\sum|\mathbf{y}|}{N}}$.

Here $N$ is the number of data points used for the identification which is equal to the length of vector $\mathbf{y}$. If the identification is perfect, $\epsilon$ is equal to one, otherwise less than one. This equation is adopted from [7] and kept more general in order to be applicable for problems with negative measurement values. However, such an evaluation concerning the identification quality allows no statement about existing deviations of the identified parameters. Therefore, for a nonlinear problem, the calculation of the standard deviation $\sigma$, according to [8], of the identified parameters is included in the identification

$\sigma=\sqrt{\operatorname{diag}(\operatorname{Cov}(\hat{\theta}))}$,

with the covariance matrix

$\operatorname{Cov}(\hat{\theta})=\frac{1}{N-N_{p}} \cdot \mathbf{e}(\mathbf{X}, \hat{\theta})^{T} \cdot \mathbf{e}(\mathbf{X}, \hat{\theta}) \cdot\left(\mathbf{J}^{T} \mathbf{J}\right)^{-1}$.

The number of identified parameters is denoted by $N_{p} ; \mathbf{J}$ is the Jacobian given by

$\mathbf{J}=\frac{\partial\left[\mathbf{w}_{\mathbf{r}} \cdot \mathbf{e}(\mathbf{X}, \hat{\theta})\right]}{\partial \hat{\theta}}$.

For a linear problem the standard deviations of the identified parameters are calculated according to [9].

\subsection{Pressure-sinkage test}

The quantity describing the relationship between the penetration depth of a plate into soil and its reaction is pressure. Therefore, the measured force has to be transformed into the required quantity, since the Bevameter is equipped with a force/torque-sensor:

$p_{m}=\frac{F_{m}}{A}$. 
The "measured" pressure $p_{m}$ is calculated by the measured force $F_{m}$ acting perpendicular to the penetration plate with its area $A$. The relationship between pressure and penetration depth is either given by the Bekker equation ([10])

$p=\left(\frac{k_{c}}{b}+k_{\phi}\right) \cdot z^{n}$

or by the Bernstein equation ([11])

$p=k \cdot z^{n}$.

In both equations $z$ denotes the penetration depth and $n$ the soil deformation exponent. The soil deformation modulus $k$ in Eq. 8 is replaced by a combination of $b, k_{c}$ and $k_{\phi}$ in Eq. 7. Here $b$ is either the width of a rectangular plate or the radius of a round plate, $k_{c}$ the cohesive modulus of deformation and $k_{\phi}$ the frictional modulus of deformation. Eq. 8 is used when tools with the same plate area, but with different shapes, have to be compared against each other. Applying Eqs. 6 and 7 to Eq. 1 leads to the following objective function for soil parameter identification

$\min _{\hat{\theta}} \frac{1}{2}\left\|\mathbf{w}_{\mathbf{r}} \cdot\left[\mathbf{p}_{\mathbf{m}}-\mathbf{p}(\mathbf{X}, \hat{\theta})\right]\right\|^{2}$.

Here the vector of the identified parameters is composed by

$\hat{\theta}=\left[k_{c}, k_{\phi}, n\right]^{T}$

and the input matrix by the different plate sizes and measured penetration depths

$\mathbf{X}=\left[\begin{array}{ll}b_{1} & z_{1} \\ \vdots & \vdots \\ b_{N_{s}} & z_{N_{s}}\end{array}\right]$

For solving Eq. 9, data of at least two pressure-sinkage tests with penetration plates having a comparable shape and different dimensions are needed. Furthermore, a certain number of test repetitions leads to a more stable identification result, since the soil preparation and the soil itself introduce some scatter in the measurements. The same number of repetitions for each test results in an equal influence of all used plates on the identified parameters. For each single test a certain number of data points, each consisting of the sinkage $z_{i}$, the plate dimension $b_{i}$ and the measured force $F_{i}$ perpendicular to the penetration plate, are taken. Those data points are equidistantly spaced over the measured penetration depth. For example, for the identification with two different plates, ten tests for each plate and 100 data points for a single test are taken. This leads to 2000 samples, $N_{s}$, for soil parameter identification.

If the range of pressure is not equal for each test, the identification results are influenced in a stronger way by tests with a higher maximum pressure. This is the case among other things if tests from penetration tools with different areas for the same range of penetration depth are used. Therefore, a weighting factor $\mathbf{w}_{\mathbf{r}}$ is considered for identification. For a similar weighting of each single test it is suggested to normalize the pressure data with the max- imum pressure of each single test by setting the reciprocal of this value in the weighting vector. This means that pressure values of each single test vary between zero and one. Therefore, the influence of the amount of the measured pressure for different penetration tools is avoided. The standard deviations of the soil parameters can be calculated according to the method given in Eq. 3 to Eq. 5. This leads to a more realistic soil description, since the soil has some random characteristic. The variation of the soil parameters are given by the standard deviations. This can be considered in rover performance simulations. Furthermore, the standard deviations indicate the repeatability of tests. The described identification method using the Bekker equation (Eq. 7) is also applicable for the Bernstein equation (Eq. 8).

The proposed method solves the problem of an improper excessive weighting of values at low sinkage as it is the case in standard soil parameter identification, as described in [12]. In these algorithms the logarithm of the pressure and sinkage is taken and the identification is transformed into a linear regression. However, this does not represent the original identification problem. To overcome this problem, Wong proposes a weighting with the square of the pressure for the logarithmized values. A comparison between the identification method proposed by Wong and the method proposed in this paper is given in Fig. 3. The Bernstein equation, Eq. 8, is used for identification. Both methods lead to very good identification results. However, it can be seen that the proposed algorithm is better (see the values given for $\epsilon$ ).

\subsection{Shear test}

In case of the determination of the shear parameters the measured torque has to be converted into shear stress. Therefore the equation proposed in [13] is used

$\tau=\frac{3 \cdot T_{m}}{2 \pi\left(r_{o}^{3}-r_{i}^{3}\right)}$.

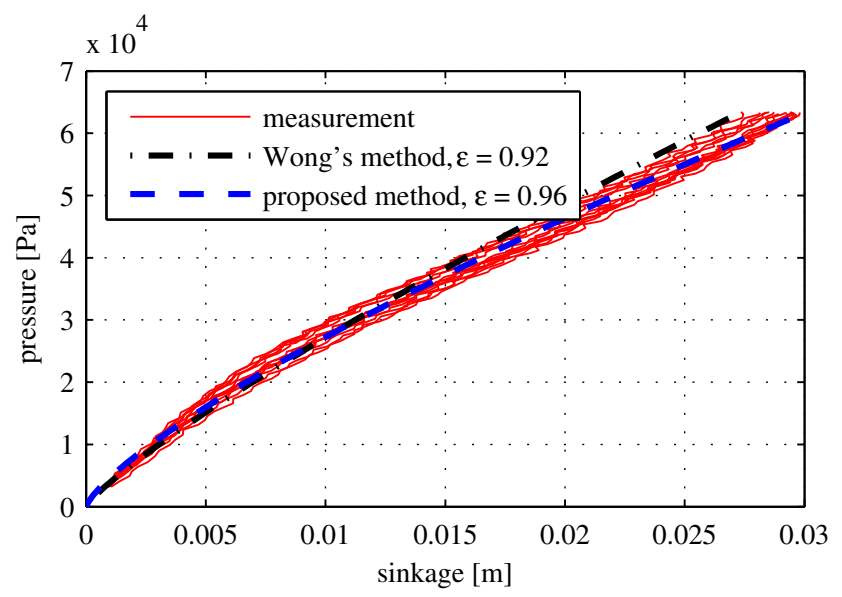

Fig. 3. Comparison between Wong's identification method and the proposed method. 
Table 1

Criteria for evaluation the preparation methods.

\begin{tabular}{ll}
\hline Criterion & Description \\
\hline 1 & $\begin{array}{l}\text { The preparation method has to be applicable for a small } \\
\text { soil bin to evaluate soil samples as well as for a testbed } \\
\text { dedicated for rover tests. For the measurements presented } \\
\text { in this paper the volume of the soil bin is } 90 \mathrm{~L} .\end{array}$ \\
& $\begin{array}{l}\text { The soil preparation has to be performed by several } \\
\text { persons according to a given procedure without leading to } \\
\text { any remarkable differences in the recorded measurements. }\end{array}$ \\
3 & $\begin{array}{l}\text { The value for } \epsilon \text {, see Eq. } 2 \text {, has to be as close to one for the } \\
\text { identification of the pressure-sinkage test. This value } \\
\text { indicates the quality of the identification. }\end{array}$ \\
\hline
\end{tabular}

The measured torque is denoted by $T_{m}$ and the shear stress by $\tau$. The tool dimensions are given by the inner and outer radius of a shear ring $\left(r_{i}, r_{o}\right)$. The soil shear stress-strain curve is described by the equation given in [13]

$\tau=(c+\sigma \tan \phi) \cdot\left(1-e^{\frac{-j}{K}}\right)$.

The soil parameter $c$ denotes the cohesion, $\phi$ the internal friction angle and $K$ the soil deformation modulus. Input parameters are the normal pressure $\sigma$, which is identical to the normal pressure $p$ in Section 2.1, and the soil deformation $j$ calculated from the shear angle $\alpha$ and the tool dimensions:

$j=\frac{r_{o}+r_{i}}{2} \cdot \alpha$.

By applying Eqs. (12) and (13) to Eq. 1 an identification of the soil shear parameters is possible. Shear tests at different normal pressures have to be performed, since the shear stress is dependent on the normal pressure. The weighting factor can be calculated according to the method given in Section 2.1. The standard deviations of the shear parame- ters can be calculated according to the method given in Eq. 3 to Eq. 5, too.

In most cases it is sufficient to determine the cohesion and the internal friction angle. It is assumed that the shear motion is steady-state after a certain rotation angle, i.e. there is no change in shear stress even if the shear motion continues. If this takes place, Eq. 13 is simplified to the following expression commonly known as Mohr-Coulomb failure criterion

$\tau=c+\sigma \tan \phi$.

Applying Eqs. (12) and (15) to Eq. 1 leads to a linear minimization problem, which can be solved by the ordinary linear least squares method to determine the values for the cohesion and the internal friction angle from measurement data of the steady-state part. The standard deviations can be calculated according to [9].

\section{Test results}

The results of the Bevameter tests, both for pressuresinkage tests and shear tests, are given in this section.

\subsection{Pressure-sinkage tests}

With the soil parameter identification methods given in the above section it is possible to characterize the analysed soils by means of reliable values according to the chosen pressure-sinkage relationship. Before starting a measurement campaign to identify the influence of the test setup on the soil parameters, an applicable soil preparation method leading to reproducible and reliable measurement results has to be found first.

\subsubsection{Soil preparation tests}

The soil preparation method has to be in line with the three criteria given in Table 1.

Table 2

Description of investigated preparation methods.

\begin{tabular}{|c|c|c|c|c|c|c|c|c|c|c|c|}
\hline \multirow{2}{*}{$\begin{array}{l}\text { Preparation } \\
\text { method }\end{array}$} & \multicolumn{3}{|c|}{ Loosening tool } & \multicolumn{3}{|c|}{ Levelling tool } & \multicolumn{2}{|c|}{ Loosening pattern } & \multicolumn{2}{|c|}{ Loosening movement } & \multirow{2}{*}{$\begin{array}{l}\text { Loosening } \\
\text { depth }(\mathrm{cm})\end{array}$} \\
\hline & $\begin{array}{l}\text { Little } \\
\text { rake }\end{array}$ & $\begin{array}{l}\text { Hand } \\
\text { shovel }\end{array}$ & $\begin{array}{l}\text { Garden } \\
\text { rake }\end{array}$ & $\begin{array}{l}\text { Little } \\
\text { rake }\end{array}$ & $\begin{array}{l}\text { Hand } \\
\text { shovel }\end{array}$ & $\begin{array}{l}\text { Levelling } \\
\text { board }\end{array}$ & Parallel & Crosswise & $\begin{array}{l}\text { Straight- } \\
\text { forward }\end{array}$ & $\begin{array}{l}\text { Raking in } \\
\text { small steps }\end{array}$ & \\
\hline 2 & $\mathrm{X}$ & & & & & $\mathrm{X}$ & $\mathrm{X}$ & & & $\mathrm{X}$ & 8 \\
\hline 3 & $\mathrm{X}$ & & & $\mathrm{X}$ & & & $\mathrm{X}$ & & $\mathrm{X}$ & & 8 \\
\hline 4 & $\mathrm{X}$ & & & $\mathrm{X}$ & & & $\mathrm{X}$ & & & $\mathrm{X}$ & 8 \\
\hline 7 & $\mathrm{X}$ & & & $\mathrm{X}$ & & & & $\mathrm{X}$ & $\mathrm{X}$ & & 8 \\
\hline 8 & $\mathrm{X}$ & & & $\mathrm{X}$ & & & & $\mathrm{X}$ & & $\mathrm{X}$ & 8 \\
\hline 9 & & $\mathrm{X}$ & & & & $\mathrm{X}$ & Shovel is & digged in, $\mathrm{s}$ & and is liftec & d trickled down & 12 \\
\hline 10 & & $\mathrm{X}$ & & & $\mathrm{X}$ & & from a $\mathrm{c}$ & onstant heig & ht $(7 \mathrm{~cm})$ & & 12 \\
\hline 11 & $\mathrm{X}$ & & & & & $\mathrm{X}$ & $\mathrm{X}$ & & $\mathrm{X}$ & & 13 \\
\hline 12 & $\mathrm{X}$ & & & & & $X$ & $\mathrm{X}$ & & & $\mathrm{X}$ & 13 \\
\hline
\end{tabular}


Different soil preparation methods were identified to be evaluated for the soil preparation. An overview of them is listed in Table 2. They are tested on a dry quartz sand (soil01) using the tools shown in Fig. 4. Information about the grain size distribution, and the bulk density, $\rho$, of the soils used for the Bevameter tests, is given in Fig. 5. All three soils are dry, frictional and granular. Soil02 is a mixture of two components: olivine and quartz sand. The grain sizes of both components are different. This is indicated by the grain size distribution of soil02, where about $65 \%$ of the soil weight has a particle size lower than $0.2 \mathrm{~mm}$ caused by the olivine. The remaining $35 \%$ represent the quartz portion. Soil08 is fire clay, which is used for special cases (trapped situation) in rover mobility investigations.

The methods vary, based on the soil loosening tools used and the handling of these tools, e.g. crosswise or parallel raking. The best three methods determined by the evaluation are selected for a further investigation on a different soil (soil02). The results of the evaluation are shown in Table 3. The handling of the tools for the rejected preparation methods is more complex than for the selected

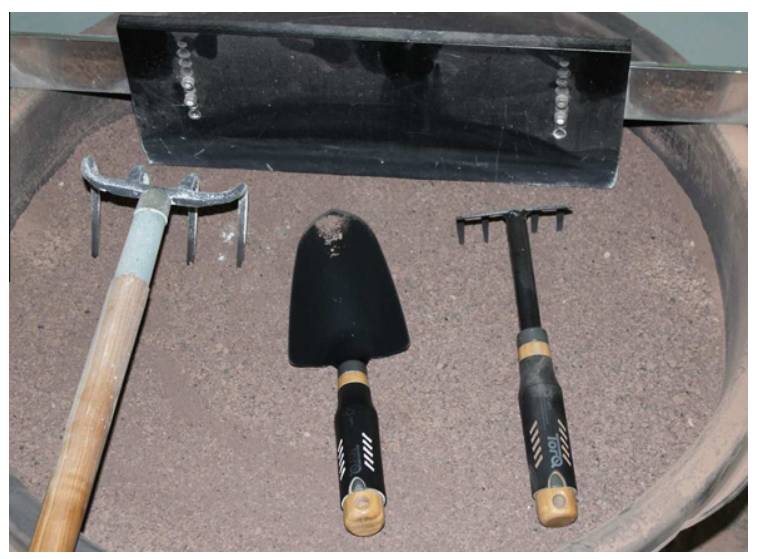

Fig. 4. Tools used for soil preparation method testing; levelling board (top), garden rake (left), hand shovel (middle) and little hand rake (right).

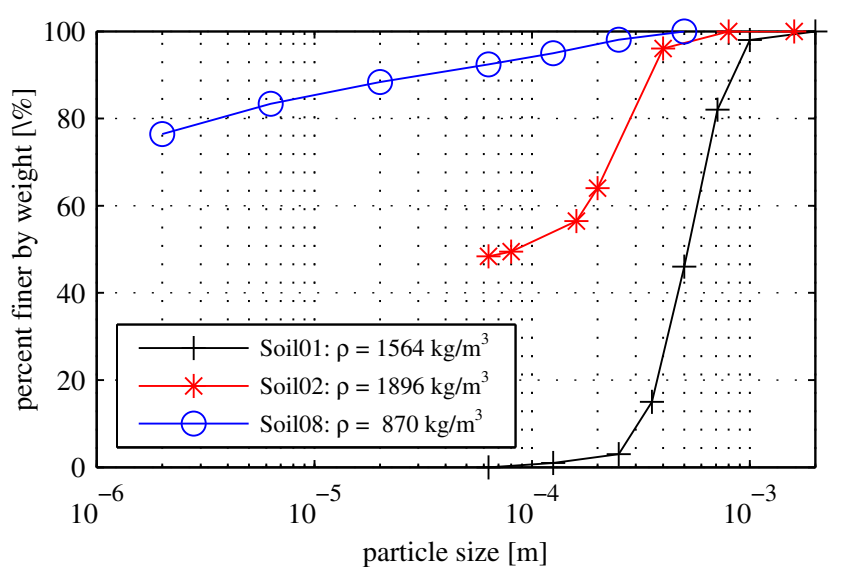

Fig. 5. Grain size distribution of the used soils for the Bevameter tests.
Table 3

Evaluation of preparation method according to the given criteria.

\begin{tabular}{cccccccccccccc}
\hline \multicolumn{11}{c}{ Preparation method } & \multicolumn{11}{c}{} & 1 & 2 & 3 & 4 & 5 & 6 & 7 & 8 & 9 & 10 & 11 & 12 & 13 \\
\hline crit 1 & - & - & - & - & - & - & - & - & + & - & + & - & + \\
crit 2 & + & + & + & + & + & + & + & + & - & - & + & + & + \\
crit 3 & - & - & - & - & - & - & - & - & + & - & + & - & + \\
$\sum$ & -1 & -1 & -1 & -1 & -1 & -1 & -1 & -1 & 1 & -3 & +3 & -1 & +3 \\
\hline
\end{tabular}

methods. These methods include certain sequences of different preparation movements as well as a certain preparation pattern. For some of the rejected methods, the soil loosening depth is too small. Therefore, these methods are not applicable to a large testbed, since they are time consuming and very difficult in handling or they do not completely loosen the soil to get rid of undesired precompactions. Nevertheless, they are tested to avoid the neglecting of any possible preparation method. The soil loosening tools for the selected preparation methods $(9,11 \& 13)$ can be increased in dimensions and are hence possible tools for the use in a large soil bin. A lesson learned from first tests is that an accurate instruction how to use the soil preparation tools is indispensable. However, when using shovels for soil mixing the results show that the possibility of diverging measured pressure-sinkage curves for such a preparation is clearly larger compared to preparing the soil with a rake. Criterion 3 is evaluated according to the quality of the identification for all tested methods, see Fig. 6 .

Based on the results of the first evaluation the best three methods are selected and tested on a different soil. Since criterion one and criterion two is not effected by the soil, only criterion three is investigated, see Fig. 6. It is evident that preparation method 13 is, in general, the best method for soil preparation. Therefore, at all further investigations the soil is prepared according to this method.

\subsubsection{Penetration velocity tests}

Although there are some approaches to include the penetration velocity in the Bekker equation [14], there is almost no information available about its influence on the pressure-sinkage relationship. Therefore, the capability of the developed Bevameter to control the penetration velocity is used and tests at different sinkage speeds on different soils are performed. For each soil and each penetration velocity three different circular plates with the radii $0.025,0.05$ and $0.075 \mathrm{~m}$ are used. These dimensions span the expected contact area of wheels for rovers up to the size of the planned ExoMars rover. The tests are performed at three different penetration velocities, $0.48 \mathrm{e}-3,2.4 \mathrm{e}-3$ and $4.8 \mathrm{e}-3 \mathrm{~m} / \mathrm{s}$. These velocities are the intersection of the maximum possible penetration velocity of the used Bevameter and low speeds of the ExoMars breadboard. Each test is repeated twelve times.

During the evaluation of these tests, an influence on the penetration velocity is observed. The smaller the mean grain size of a soil (e.g. soil08), the stiffer the pressure-sink- 

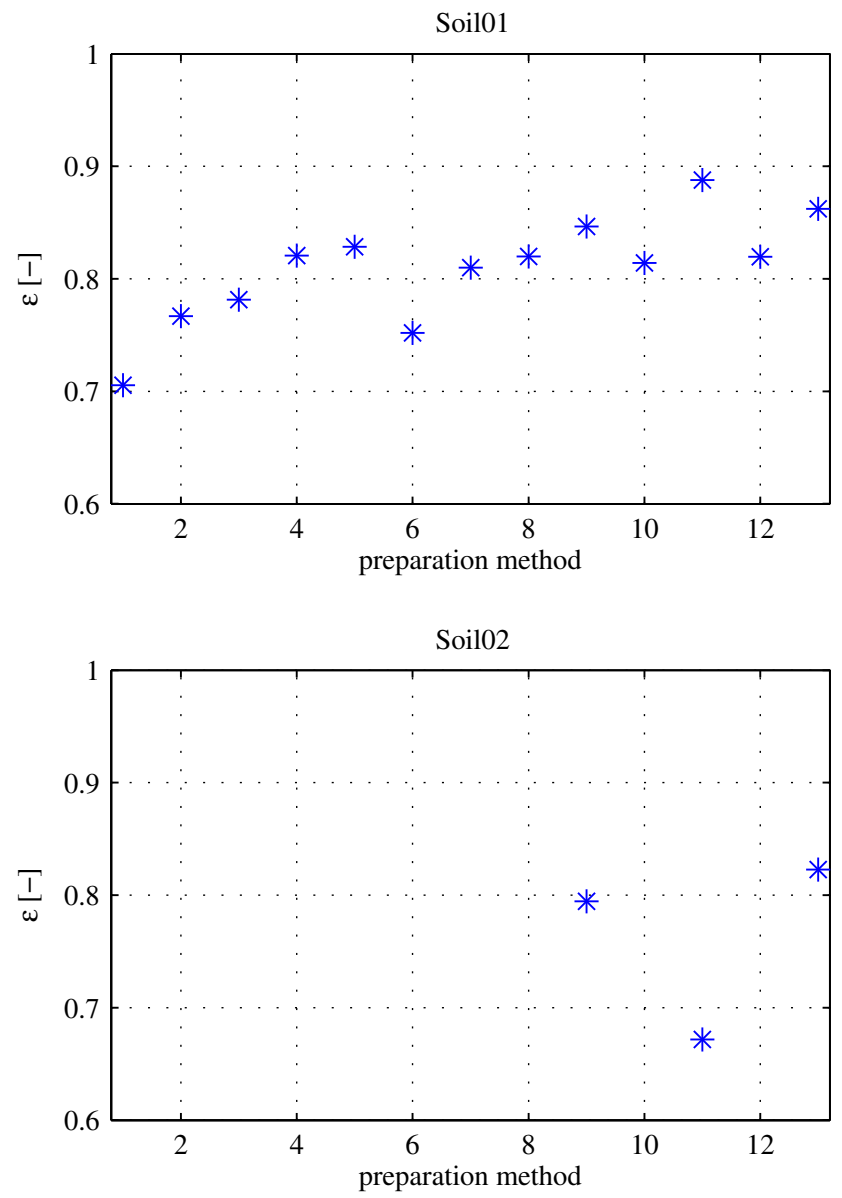

Fig. 6. Quality of identification for different preparation for soil01 (dry quartz sand) and soil02. age relationship for lower velocities. This is shown clearly in measurement results for tool02 in Fig. 7. Information about the soils used is shown in Fig. 5. Since a part of the soils used for the rover performance testing and validation of wheel-soil contact models are similar to soil02 and soil08, the determination of the Bekker parameters have to be done very carefully with a penetration velocity, which is adequate to the average rover speed.

\subsubsection{Influence of tool shape and size}

In [12] it is recommended to use penetration tools being equivalent to the wheel print for soil parameter determination. A test series with a circular plate and rectangular plates having the same area is therefore performed. The side ratios of the rectangular plates are 1:1, 1:3, 1:5 and 1:7. Reference tools are circular plates with $0.05 \mathrm{~m}$ or $0.075 \mathrm{~m}$ radii. Penetration velocity is set to $4.8 \mathrm{e}-3 \mathrm{~m} / \mathrm{s}$. The measured pressure-sinkage curves for the soil types soil01, soil02 and soil08, for tools with an area of $0.0079 \mathrm{~m}^{2}$ (equivalent for a circular plate with $r=0.05 \mathrm{~m}$ ) are given in Fig. 8. It is observed that, for side ratios of 1:5 or greater, the pressure-sinkage relationship gets softer for soil01 and soil02.

The measured curves can be explained by the observations of the soil behaviour under a penetration plate given in $[15,16]$. At the first stage of a penetration test, pure compaction of the soil takes place. This is shown by the almost identical pressure-sinkage curves at the initial stage. In this stage soil compaction is the dominating effect under the plate. The curves are not influenced by the tool dimensions. Since soil08 is a very loose one, only compaction is
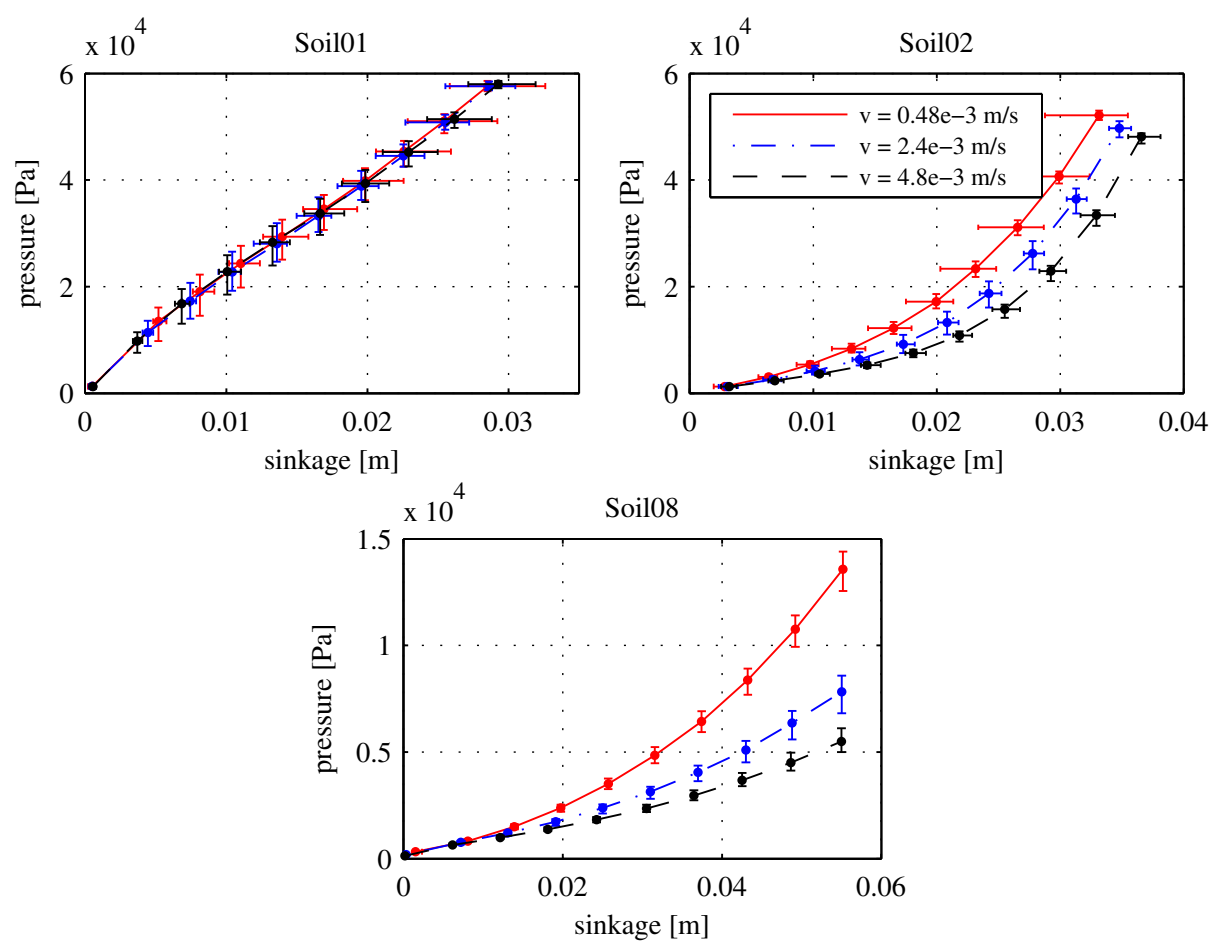

Fig. 7. Measurements of pressure-sinkage curves for three different soils at three different penetration velocities with a circular plate $(r=0.05 \mathrm{~m})$; the mean grain size for each soil gets lower from left to right; the error bars indicate the statistical spread in the measurement. 

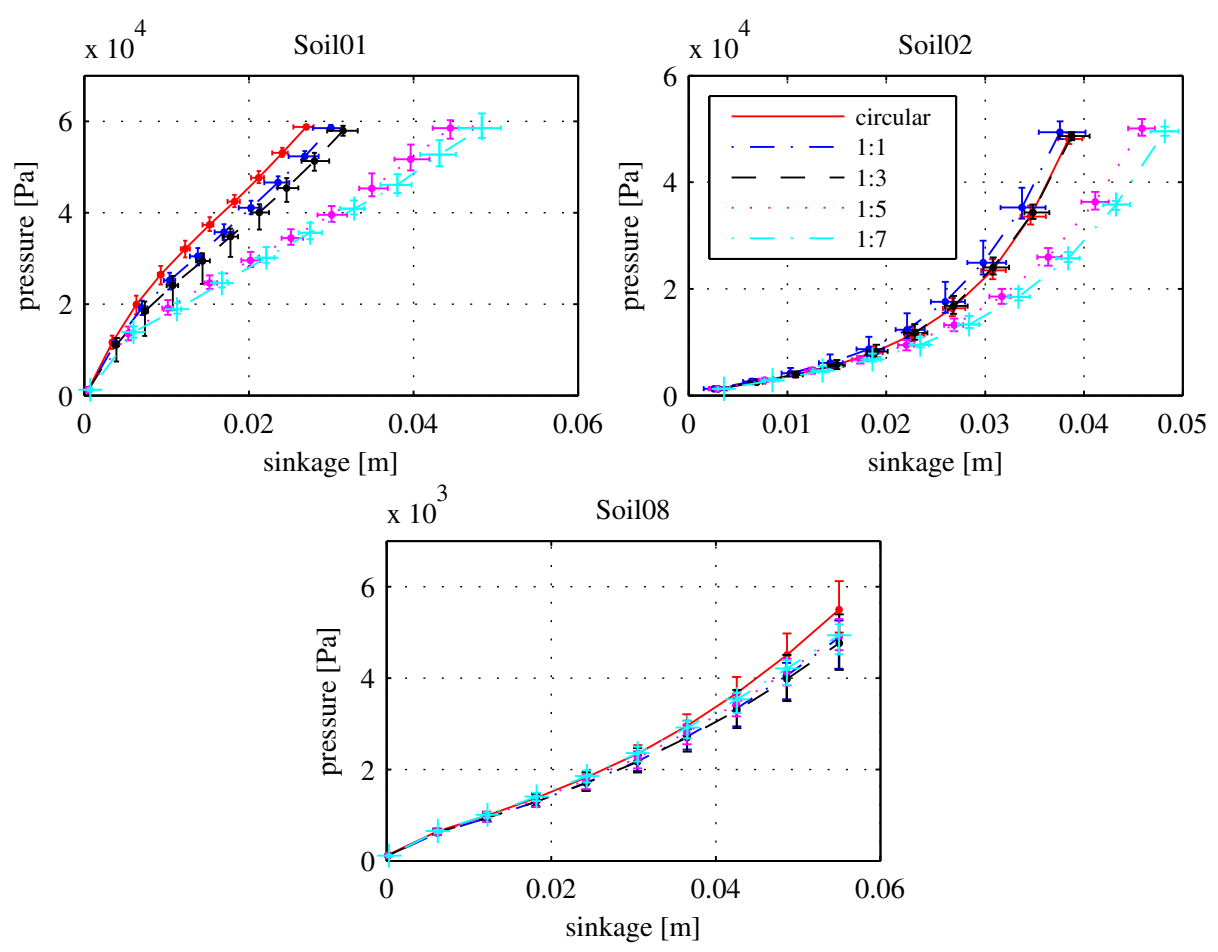

Fig. 8. Measured pressure-sinkage relationship for a circular penetration plate $(r=0.05 \mathrm{~m})$ and rectangular plates with the same area and different side ratios for different soils.

observed and the curves do not diverge in the measured range. The failure zone below the plate is rectangular during compaction if the test is regarded in cross section, see Fig. 9.

By increasing the penetration depth the lower corners of the rectangular failure zone round off and the failure zone transforms from a rectangular shape via a truncated cone to a fully formed cone. If the failure zone starts to transform towards the cone, lateral soil compression and flow also commences. At this stage the pressure-sinkage curve gets softer according to [16]. This is also visible in the measured curves for soil01 and soil02 at plate side ratios of 1:5 and 1:7. This effect is more clearly recognized for narrower plates because the transformation of the soil failure zone into a cone tends to be faster than for plates with larger

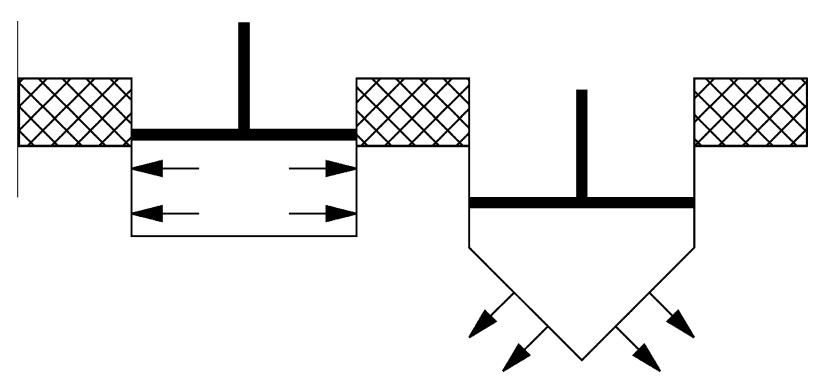

Fig. 9. Schematic 2D illustration for the soil behaviour under a pressure plate during a pressure-sinkage test; pure soil compaction with a rectangular failure zone (left); lateral soil compaction and soil flow with a cone as failure zone (right). side ratios. This effect is not observed in Fig. 8 (soil01 and soil02) for the plate with a side ratio $1: 3$ as well as for soil08. It is assumed that those curves diverge at higher sinkages. However, these sinkages are beyond the mechanical constraints of the used Bevameter. The illustration given in Fig. 9 is more suitable for soil02 and soil08. For both soils the side walls do not collapse, even for deeper penetrations. For soil01 (quartz sand) the illustration is very idealistic, since the side walls collapse for a penetration test and the soil behaves more free-flowing than the others. However, the classification of the test into a pure compression stage and a lateral soil compression stage including flow can also be seen for the quartz sand in Fig. 8. The boundary between both stages is approximately at a soil depth of 5-7 mm.

Summing up, the tool dimensions affect the measurements. To characterize the soil in terms of determining Bekker parameters for a wheel with a certain dimension the use of a circular plate with the same or almost the same size as the wheel print is adequate.

\subsection{Shear test results}

In the previous section different effects on the measured pressure-sinkage curves were given. For a complete description of the wheel-soil contact the shear parameters are also needed. Therefore, the Bevameter is used as well as for the shear measurements. The Bevameter control subsystem includes a control algorithm that adjusts the 
sinkage of the shear tool by holding the normal force constant. Only test results for soil01 and soil02 are presented, because these include enough information about the influence of the different setup parameters on the soil shear parameters.

\subsubsection{Variation of tool size}

To investigate the influence of the tool size, two tools, depicted in Fig. 10, with different dimensions, are used. The rotational velocity is set to $0.1 \mathrm{rpm}$ for both tools to reach almost steady-state conditions. An overview of the tool dimensions is given in Table 4. The results are shown in Fig. 11. It can be seen that a shear ring with a larger inner radius (tool07) leads to a higher cohesion and internal friction angle. Therefore, the appropriate tool dimensions for the soil characterization in order to get the parameters for a wheel-soil contact model cannot be determined by these tests. A further test campaign, including single wheel testing and simulation, is required to identify the appropriate tool dimensions.

\subsubsection{Variation of number of grousers}

In a further test series the influence of the number of grousers on the soil parameters is investigated. For these tests tool07, see Table 4 for its dimensions, is used. The grouser height is changed to $7.5 \mathrm{~mm}$. The rotational velocity of the tool is set to $0.1 \mathrm{rpm}$. The measurements are taken with 6,8 or 12 equally spaced grousers on the shear tool. The results are shown in Fig. 12. It is clearly visible, that the number of grousers does not affect significantly the shear stress over the measured range of normal pressure. Decreasing the number of grousers to two or even zero has certainly an effect on the measurements. However,

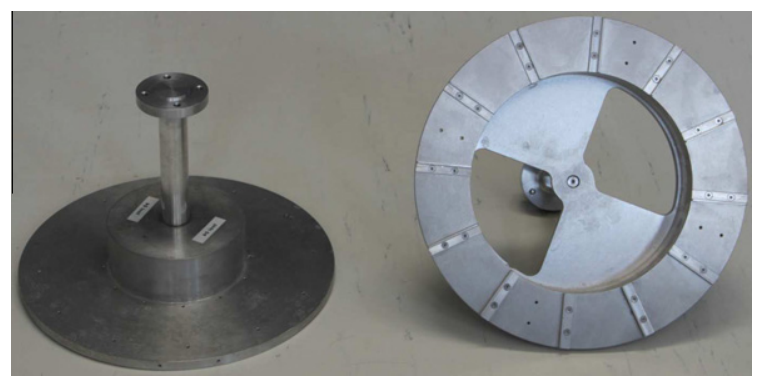

Fig. 10. Depicted shear tools, tool04 left, tool07 right.

Table 4

Dimensions of the shear tools.

\begin{tabular}{lll}
\hline & Tool04 $(\mathrm{t} 04)$ & Tool07 $(\mathrm{t} 07)$ \\
\hline Inner radius, $r_{i}(\mathrm{~m})$ & 0.05 & 0.1 \\
Outer radius, $r_{o}(\mathrm{~m})$ & 0.15 & 0.15 \\
No. of grousers $(-)$ & 12 & 12 \\
Grouser height $(\mathrm{m})$ & 0.005 & 0.005 \\
\hline
\end{tabular}
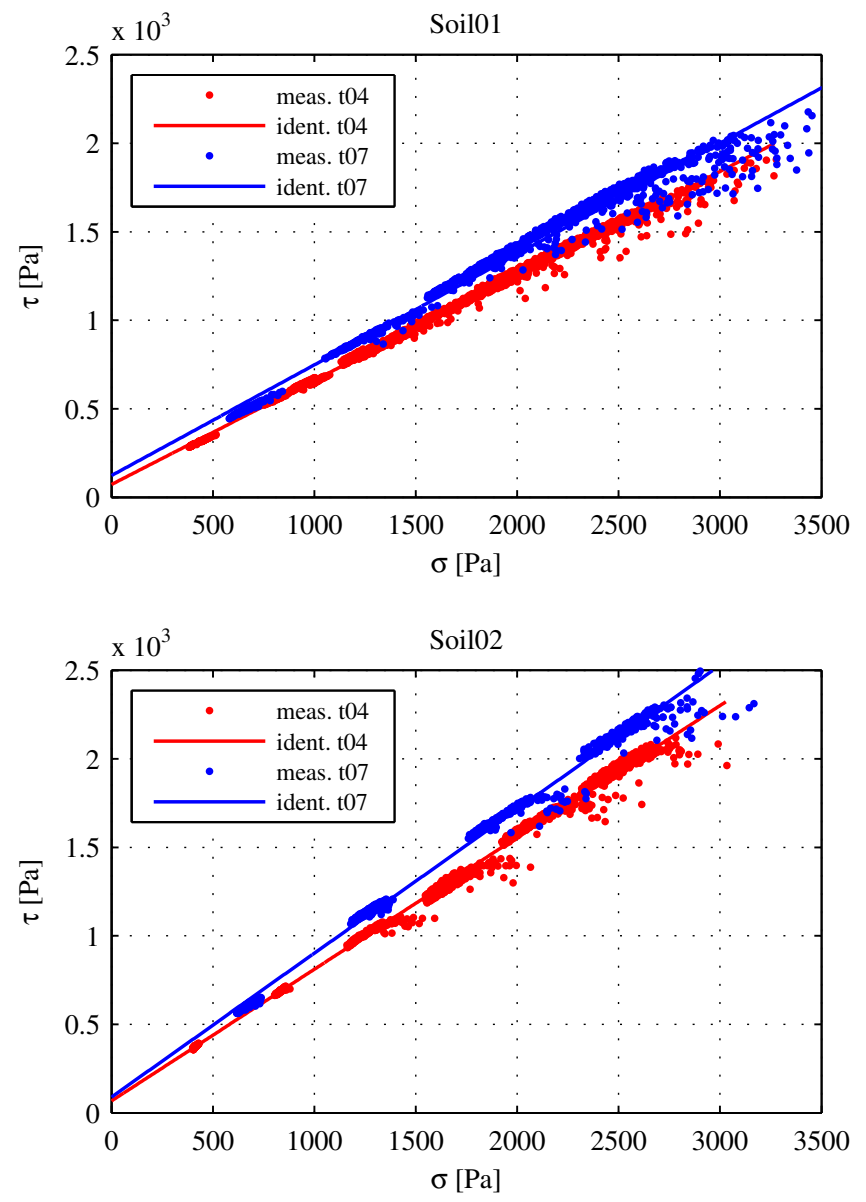

Fig. 11. Shear test results for different shear rings for two soils.

such a test setup is not a realistic representation of a wheel shear motion. Thus these test results are omitted.

\subsubsection{Variation of grouser height}

The influence of the grouser height on the soil parameters is also investigated. Tool07 is used for these tests. The number of grousers has the amount of 12 . The rotational velocity is $0.1 \mathrm{rpm}$. The grouser height is altered from $2,5,7.5$ to $10 \mathrm{~mm}$. The results are shown in Fig. 13. Increasing the grouser height leads to a higher shear stress for the same normal pressure. In Section 4.2.3 a detailed investigation in terms of shear parameter identification is given. It can be stated that for a proper soil parameter determination the usage of grousers similar to the grousers on the wheels suits best.

\subsubsection{Variation of shear velocity}

A further feature of the Bevameter used in this study is the rotational shear velocity, which can be varied and controlled. In a test series, the influence of the rotational speed on the shear parameters is investigated. Tool07 with 12 grousers and a grouser height of $7.5 \mathrm{~mm}$ is used for the tests. The rotational velocity is set to $0.1,0.2$ and 

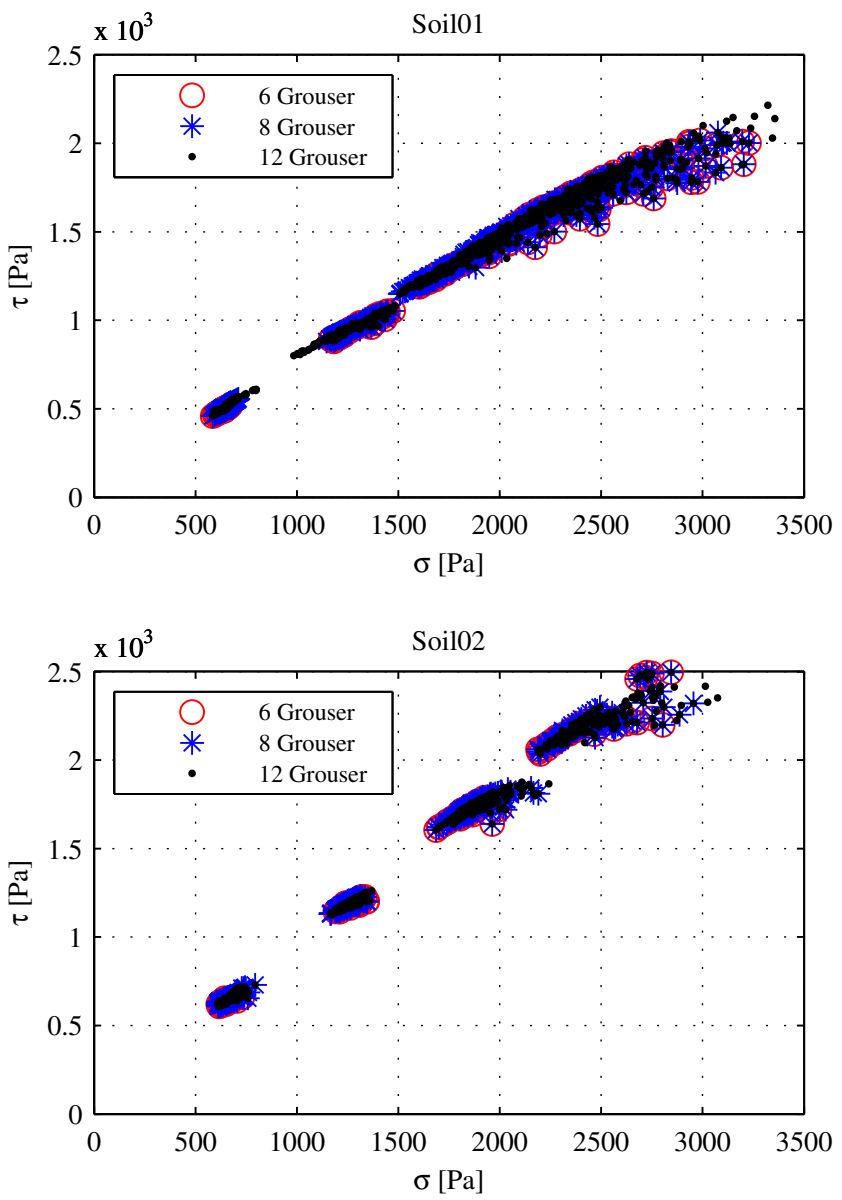

Fig. 12. Measurement data from tests with different numbers of grousers for two soils.

$0.3 \mathrm{rpm}$. The results are shown in Fig. 14. No significant influence on the shear parameters is observed by the test.

\section{Identification results}

The identified results of the test campaign measurement data are given in the following section.

\subsection{Identification for the pressure-sinkage test case}

For the identification of the pressure-sinkage tests the methods as described in Section 2.1, are used. The weighting factor is calculated according to the description given in Section 2.1.

\subsubsection{Penetration velocity tests}

The identified Bekker parameters, including their standard deviations, for the penetration velocity are given in Table 5. It can be seen that for almost all parameters the standard deviations is below $10 \%$ relative to the identified values. For parameter $n$ it is below 1\%. This result confirms the applicability of the chosen soil preparation method. The values for soil01 are almost constant for different pen-
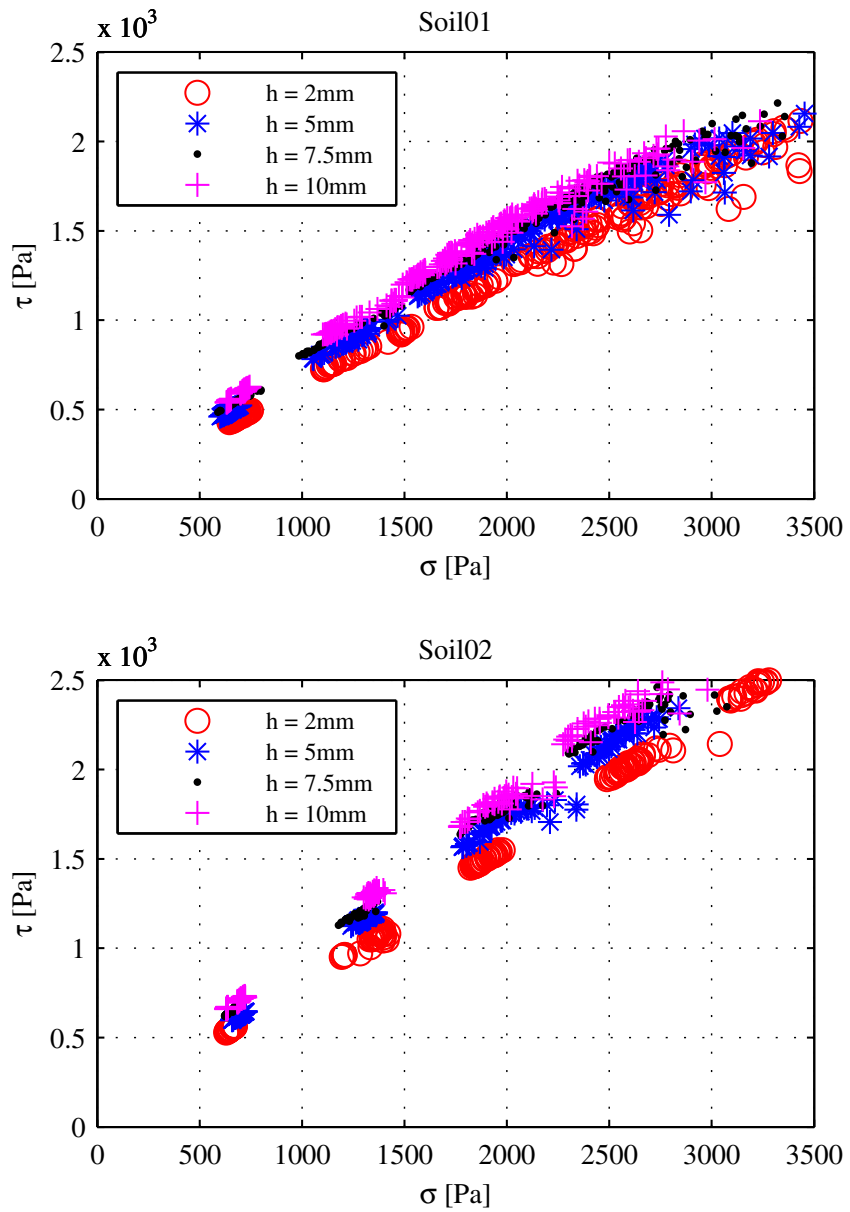

Fig. 13. Measurement data from tests with different grousers heights for two soils.

etration velocities. This is obvious, since its pressure-sinkage curves do not vary for different sinkage speeds, as shown in Fig. 7. For soil02 and soil08 the values are different. However, there is no trend identifiable, as for example an increase for the value $n$ at higher penetration velocities. This is among other things caused by the numerics of the identification algorithm. The used algorithms offer the best solution for the given minimization problem (Eq. 9). Therefore a glance at the soil parameters does not suffice for a comparison of different soils. There has to be always a comparison of the pressure-sinkage curves for a detailed analysis.

\subsubsection{Influence of tool size and shape}

To determine the influence of the tool size and shape on a mathematical representation of the pressure-sinkage relationship, Eq. 8 is applied to Eq. 1. The Bernstein equation is used here because the tool shapes are varying and the comparison of the identified parameters turns out to be easier. The identified soil parameters relative to the soil parameter of the circular plate and also the quality of the fit are depicted in Fig. 15. The fit quality is good. The val- 

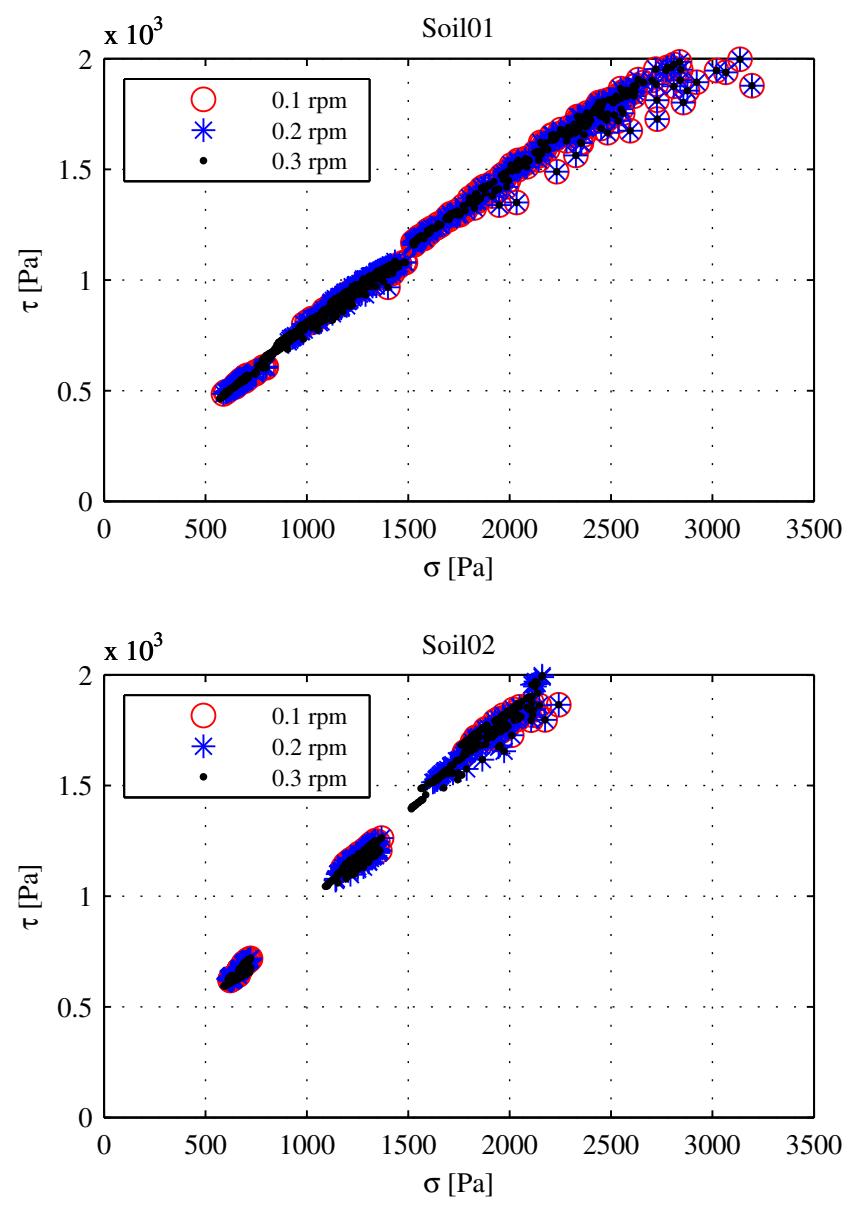

Fig. 14. Measurement data from tests with different shear velocities for two soils.

ues for the relative standard deviations are below $12 \%$ for $k$ and below $2 \%$ for $n$. The identified soil parameters $k$ and $n$ are depicted with their standard deviations. All values are relative to the parameters of the circular plate. It can be seen that there is no clear trend in the identified parameters. As mentioned above, the pressure-sinkage curves have to be considered in addition to the identified numbers to compare tests.

\subsection{Identification for the shear test case}

The identified values for the shear tests are given in this section. For shear soil parameter identification, Eq. 15 is applied to Eq. 1. The weighting factor is set to one.

\subsubsection{Variation of tool size}

As depicted in Fig. 11 the tool size has an influence on the shear measurements. The identified values with their standard deviations for the test are given in Table 6. Furthermore, the relative difference between the identified parameters are listed. For the cohesion the difference is quite high. However, due to their small effect of values around and below $100 \mathrm{~Pa}$ on the wheel performance the influence of the tool dimension on the cohesion is negligible. Moreover, the relative increase of about five to six percent in the internal friction angle influences the wheel performance much more. Mohr-Coulomb lines calculated from the identified values are plotted in Fig. 11. It can be seen that the calculated lines are matching the measurement data. A consequence of this result is that the shear ring dimensions have to be appropriate wheels for a correlation of rover performance tests and simulations for the rover.

\subsubsection{Variation of number of grousers}

The result of these tests (the measurement data is shown in Fig. 12) is that there is no recognizable influence of the number of grousers on the shear parameters. However, this statement is only valid for grouser numbers between 6 and 12. An extrapolation for a higher or lower number of grousers can of course lead to other results. The identified results and their standard deviations are given in Table 7. The values are varying less as it is expected from the measurement data.

\subsubsection{Variation of the grouser height}

The measurement data for these tests, depicted in Fig. 13, shows an influence of the grouser height. With increasing grouser height the shear stress increases. This effect can also be noticed in the identified shear parameters,

Table 5

Identified Bekker parameters and their standard deviations for different soils and different penetration velocities.

\begin{tabular}{|c|c|c|c|c|}
\hline & Soil01 & Soil02 & Soil08 & Penetration velocity $(\mathrm{m} / \mathrm{s})$ \\
\hline $\begin{array}{l}k_{c}\left(\mathrm{~N} / \mathrm{m}^{n+1}\right) \\
k_{\phi}\left(\mathrm{N} / \mathrm{m}^{n+2}\right) \\
n(-) \\
\epsilon(-)\end{array}$ & $\begin{array}{c}-9.8 e 3 \pm 4.6 e 2 \\
1.2 e 6 \pm 3.1 e 4 \\
0.80 \pm 0.01 \\
0.82\end{array}$ & $\begin{array}{c}-1.4 e 6 \pm 1.1 e 5 \\
1.0 e 8 \pm 7.0 e 6 \\
2.14 \pm 0.01 \\
0.81\end{array}$ & $\begin{array}{c}-1.5 e 4 \pm 4.6 e 2 \\
3.0 e 6 \pm 8.2 e 4 \\
1.82 \pm 0.01 \\
0.88\end{array}$ & $4.8 e-4$ \\
\hline $\begin{array}{l}k_{c}\left(\mathrm{~N} / \mathrm{m}^{n+1}\right) \\
k_{\phi}\left(\mathrm{N} / \mathrm{m}^{n+2}\right) \\
n(-) \\
\epsilon(-)\end{array}$ & $\begin{array}{c}-1.2 e 4 \pm 5.0 e 2 \\
1.2 e 6 \pm 3.3 e 4 \\
0.81 \pm 0.01 \\
0.81\end{array}$ & $\begin{array}{c}-3.2 e 7 \pm 2.0 e 6 \\
1.9 e 9 \pm 1.1 e 8 \\
3.02 \pm 0.02 \\
0.83\end{array}$ & $\begin{array}{l}7.3 e 3 \pm 1.9 e 2 \\
5.7 e 5 \pm 1.4 e 4 \\
1.57 \pm 0.01 \\
0.89\end{array}$ & $2.4 e-3$ \\
\hline $\begin{array}{l}k_{c}\left(\mathrm{~N} / \mathrm{m}^{n+1}\right) \\
k_{\phi}\left(\mathrm{N} / \mathrm{m}^{n+2}\right) \\
n(-) \\
\epsilon(-)\end{array}$ & $\begin{aligned}-9.8 e 3 & \pm 4.6 e 2 \\
1.2 e 6 & \pm 3.41 e 4 \\
0.82 & \pm 0.01 \\
0.81 & \end{aligned}$ & $\begin{array}{c}-1.8 e 8 \pm 2.2 e 7 \\
1.0 e 10 \pm 1.2 e 9 \\
3.59 \pm 0.03 \\
0.82\end{array}$ & $\begin{array}{l}7.0 e 3 \pm 1.6 e 2 \\
2.2 e 5 \pm 5.0 e 3 \\
1.45 \pm 0.01 \\
0.88\end{array}$ & $4.8 e-3$ \\
\hline
\end{tabular}



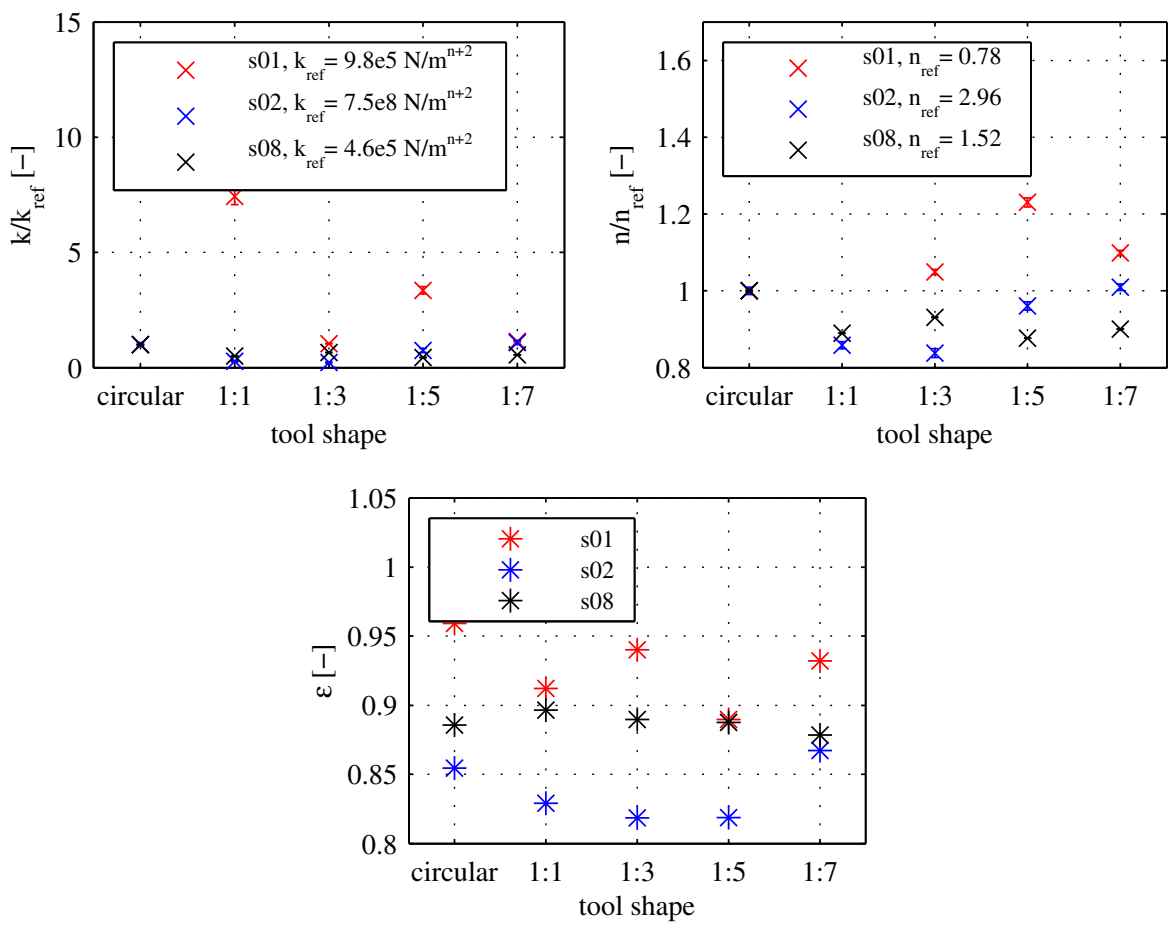

Fig. 15. Identified soil parameters and quality of the fit from tools with different tool shapes for different soils.

Table 6

Comparison of shear parameters for different shear rings and soils.

\begin{tabular}{llllll}
\hline & \multicolumn{3}{l}{ Soil01 } & & \multicolumn{2}{l}{ Soil02 } \\
\cline { 2 - 3 } \cline { 5 - 6 } & $c(\mathrm{~Pa})$ & $\phi\left(^{\circ}\right)$ & & $c(\mathrm{~Pa})$ & $\phi\left(^{\circ}\right)$ \\
\hline Tool04 & $71.9 \pm 2.2$ & $30.5 \pm 0.1$ & & $66.5 \pm 2.8$ & $36.7 \pm 0.1$ \\
Tool07 & $122.2 \pm 22.9$ & $32.1 \pm 0.1$ & & $88.3 \pm 20.1$ & $39.1 \pm 0.1$ \\
$\begin{array}{l}\text { Relative deviation } \\
(\%)\end{array}$ & 70 & 5.3 & 32.8 & 6.5 \\
\hline
\end{tabular}

Table 7

Comparison of shear parameters for different number of grousers and soils.

\begin{tabular}{llllll}
\hline & \multicolumn{2}{l}{ Soil01 } & & \multicolumn{2}{l}{ Soil02 } \\
\cline { 2 - 3 } \cline { 6 - 6 } & $c(\mathrm{~Pa})$ & $\phi\left(^{\circ}\right)$ & & $c(\mathrm{~Pa})$ & $\phi\left(^{\circ}\right)$ \\
\hline 6 grouser & $107.9 \pm 0.1$ & $33.3 \pm 0.1$ & & $131.9 \pm 16.3$ & $40.0 \pm 0.1$ \\
8 grouser & $141.2 \pm 6.7$ & $33.3 \pm 0.1$ & & $129.7 \pm 9.3$ & $40.1 \pm 0.1$ \\
12 grouser & $128.0 \pm 4.6$ & $33.1 \pm 0.1$ & & $137.0 \pm 6.5$ & $39.8 \pm 0.1$ \\
\hline
\end{tabular}

see Fig. 16. An increase in grouser height leads to an increase in cohesion and internal friction angle. A possible explanation of this effect is that for the transformation of the measured torque into the shear stress, a vertical shear surface at the side of the ring has to be considered in addition. For the calculation of the shear stress (Eq. 12), given in [13], only the shear area of the shear ring is taken into account. However, there is a certain soil to soil friction on the side of the shear rings. This area depends on the grouser height. First investigations show that including this area is a promising approach to describe the observed effects. The high standard deviations of the cohesion can
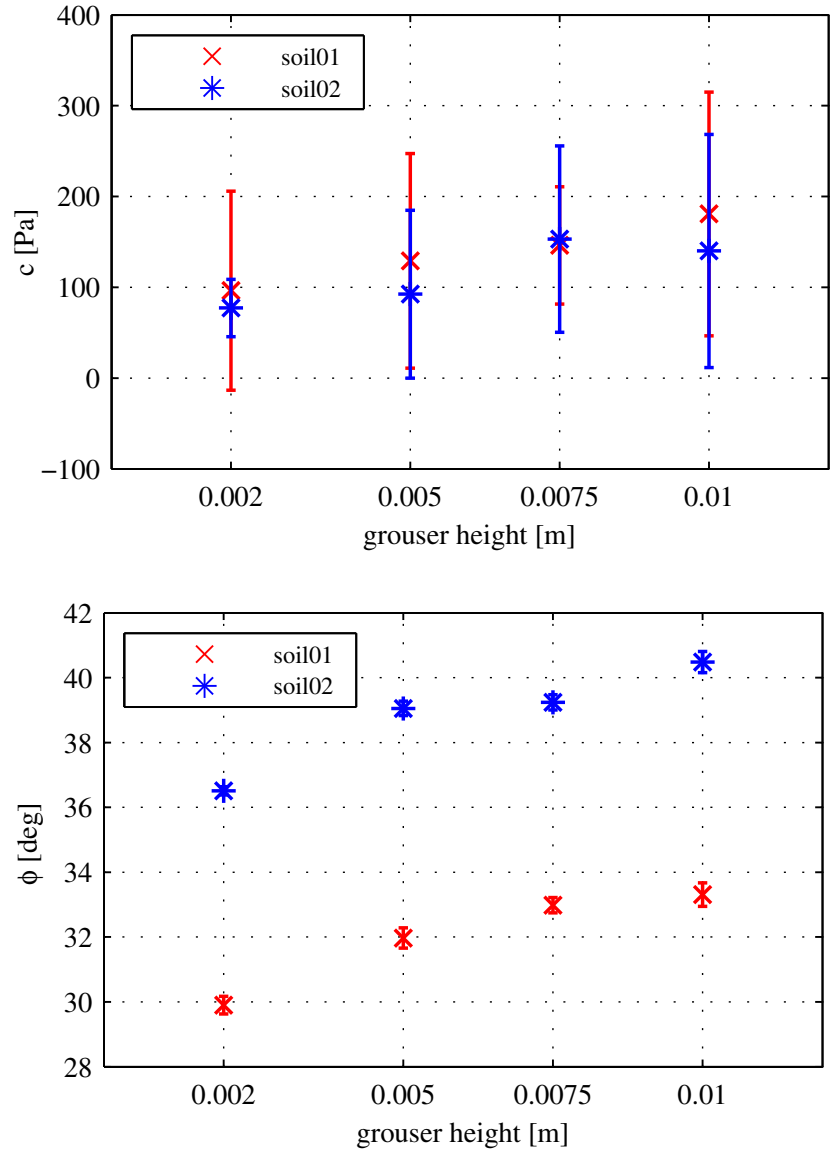

Fig. 16. Identified shear parameters including their standard deviations for different grouser heights. 
be neglected, since the influence of the cohesion on wheelsoil interaction is minor for these values.

\subsubsection{Variation of shear velocity}

The measurement data, depicted in Fig. 14, shows, that the shear velocity does not significantly influence the measurement and thus the identified shear parameters. However, an extrapolation of this statement for shear velocities lower than $0.1 \mathrm{rpm}$ or higher than $0.3 \mathrm{rpm}$ has to be regarded with care. The identified shear parameters are indicated in Table 7 for 12 grousers. The rotational velocity of the measurement data for these identified values is $0.1 \mathrm{rpm}$. The measurement data is the same as used for the shear parameter identification to compare the influence of the number of grousers.

\section{Conclusion}

An objective function for a proper soil parameter identification is presented as well as a calculation of the standard deviation of identified parameters. Combining the presented objective function with a nonlinear solver leads to improved and reliable identification results. Furthermore, the influence of the Bevameter test setup is investigated and an appropriate soil preparation method applicable for a large testbed is determined. The test results show that there are certain influences on the pressure-sinkage relationship for different penetration velocities and different penetration tool dimensions. It is also shown that the shear parameters depend on the dimensions of the shear tool and the grouser height, whereas they are invariant against the number of grousers and the rotational velocity. However it has to be stated that all results are only applicable for the presented soils or similar ones and cannot be extrapolated to broader ranges than the investigated ones without some care. It has to be remarked that the results of the shear test do not diverge for different types of soils. Future work is to find appropriate equations taking the tool dimensions, both for pressure-sinkage tests and shear tests including the grouser height into account. Moreover, this work can help to find the appropriate test setup for a validation of a soil contact model for a certain wheel at defined operating conditions.

\section{References}

[1] Gibbesch A, Krenn R, Herrmann F, Schäfer B, Rebele B, Allouis E. Multi-body system and contact simulation within the design development of planetary surface exploration systems. In: iSairas. Sapporo, Japan; 2010.

[2] Krenn R, Hirzinger G. SCM a soil contact model for multi-body simulations. In: 11th European regional conference of the international society of terrain-vehicle systems. Bremen, Germany; 2009.

[3] Apfelbeck M, Kuß S, Wedler A, Gibbesch A, Rebele B, Schäfer B. A novel terramechanics testbed setup for planetary rover wheel-soil interaction. In: 11th European regional conference of the international society of terrain-vehicle systems. Bremen, Germany; 2009.

[4] Wong JY. Data processing methodology in the characterization of the mechanical properties of terrain. J Terrmech 1980;17:13-41.

[5] Oravec HA. Understanding mechanical behavior of lunar soils for the study of vehicle mobility. Ph.D. thesis. Cleveland (OH, USA): Case Western Reserve University; 2009.

[6] Jarre F, Stoer J. Optimierung. Heidelberg,Germany: Springer Verlag; 2004.

[7] Wong JY. Measurement and characterization of the pressure-sinkage relationship for snow obtained using a Rammsonde and a Bevameter. Tech. Rep. Contract Report 72/90. Ralston (Alberta, Canada): Defense Research Establishment Suffield; 1990.

[8] Seber GAF, Wild CJ. Nonlinear regression. New York (USA): Wiley Intersciences; 2003.

[9] Papula L. Mathematik für Ingenieure und Naturwissenschaftler Band 3, 5th ed., Wiesbaden (Germany): Vieweg + Teubner; 2008.

[10] Bekker MG. Introduction to terrain-vehicle systems. Ann Arbor (MI, USA): University of Michigan Press; 1969

[11] Bernstein R. Probleme einer experimentellen Motorpflugmechanik. Der Motorwagen 1913;16:199-206.

[12] Wong JY. Terramechanics and off-road vehicle engineering. 2nd ed. Oxford (UK): Elsevier; 2010.

[13] Janosi Z, Hanamoto B. The analytical determination of drawbar pull as a function of slip for tracked vehicles in deformable soils. In: 1st International conference of the international society of terrain-vehicle systems, Turin, Italy; 1961.

[14] Grahn M. Einfluß der Fahrgeschwindigkeit auf die Einsinkung und den Rollwiderstand von Radfahrzeugen auf Geländeböden. Ph.D. thesis. Hamburg (Germany): Universität der Bundeswehr; 1996.

[15] Leis R. Soil-value system as determined with a precision Bevameter. Tech. Rep. ORA Project 03026. Ann Arbor (MI, USA): University of Michigan; 1961.

[16] Earl R, Alexandrou A. Deformation processes below a plate sinkage test on sandy loam: experimental approach. J Terramech 2001;38:153-62. 